\title{
Morphological Aspects of the Pathogenesis of Gallbladder Cholesterosis
}

\author{
Morozov IA ${ }^{1 *}$ and Khomeriky SG $^{2}$ \\ ${ }^{1}$ Chumakov Federal Scientific Center for Russian Academy of Sciences, Russia \\ ${ }^{2}$ Loginov Moscow Clinical Scientific Center, Russia
}

ISSN: 2637-7632

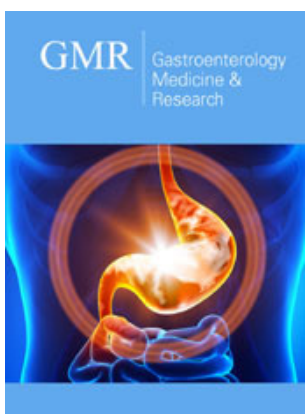

*Corresponding author: Morozov IA, Chumakov Federal Scientific Center for Russian Academy of Sciences, Russia

Submission: 地 June 06, 2019

Published: 䟧June 17, 2019

Volume 3 - Issue 3

How to cite this article: Morozov I, Khomeriky S. Morphological Aspects of the Pathogenesis of Gallbladder Cholesterosis Gastro Med Res. 3(3). GMR.000562. 2019. DOI: $10.31031 /$ GMR.2019.03.000562

Copyright@ Morozov IA,This article is distributed under the terms of the Creative Commons Attribution 4.0 International License, which permits unrestricted use and redistribution provided that the original author and source are credited.

\begin{abstract}
The article presents a new view on the pathogenesis of cholesterosis of the gallbladder, formed on the basis of the morphological study of a large number of gallbladders after cholecystectomy using modern.

Methods: Light and electron microscopy, immunohistochemistry. Essentially new information is given on the structure of the gallbladder wall and its pathomorphosis in the dynamics of the disease. The phenomenon of endocytosis absorption of bile micelles by absorptive epithelial cells and their penetration into their own plate is described for the first time. It is this process that triggers the activation of histiocytes and their transformation into tissue macrophages. For the first time, using immunohistochemistry, it was proved that only macrophages are the phenotypic precursor of foam cells.
\end{abstract}

Conclusion: The etiological factor of cholesterosis is general disorders of the body's lipid metabolism and the following changes in the metabolizing properties of absorbent biliary epithelium cells, as well as the synthetic and secretory functions of tissue macrophages.

Keywords: Gallbladder; Cholesterosis; Pathogenesis

\section{Introduction}

First messages about cholesterosis gall bubble (GC - gallbladder cholesterosis) appeared still one and a half century ago R Wirhov; A Bottcher. At the beginning of the twentieth century L Aschoff, then B Moynihan supplemented the information about this disease. It was later established Boyd [1] that inclusions in the gallbladder wall, detected by the so-called "strawberry" gall bladder, contain cholesterol esters. However, so far gallbladder cholesterosis (GC) is still insufficiently studied and little known to practitioners, which created an idea of it as a rare and difficult to diagnose disease. In the literature, CGD is described under various names: "strawberry", "raspberry", "honey", "speckled", "fish", yellow acanthosis, cholesterol polyposis, lipidosis, lipoid cholecystitis, xanthoma cholecystitis, cholesterosis, etc. The term "cholesterosis" was proposed in 1925 godu N. Men den-Sanchez and is currently the most common in the literature. A significant contribution to the study of the problem of cholesterosis of the gallbladder was made by Russian scientists - Permyakov [2]. Their book contains not only general characteristics, but also various macro- and microscopic forms, the original classification, combined pathology, as well as the results of histochemical studies and the biochemical characteristics of bile with cholesterosis. The interpretation results obtained by the authors corresponded to the level of knowledge of that time, but in many respects anticipated the interpretation of controversial points of the pathogenesis of the disease.

In spite of the considerable time spent on the study of CID, many questions concerning the prevalence, pathogenesis, diagnosis, clinical course, treatment, and management of patients remain open and are often controversial. Interest in the problem of CPD has especially increased in the last decade, when it was possible to uncover some pathogenetic mechanisms of the disease, more informative diagnostic methods have emerged, which made it possible to develop management tactics for such patients and treatment methods [3]. As before, the key issue of the pathogenesis of CGD requires an explanation - why under some conditions a violation of cholesterol metabolism leads to the formation of gallstones in the bladder, others to the development of cholesterosis proper in its wall, and thirdly, both pathological processes are identified. 
Since Virchov R, who first described this disease as early as 1857 , the specific pattern of the internal surface of the gallbladder is the presence of multiple formations (inclusions) of various shades of yellow. The size of these formations and their appearance can vary greatly, but in most cases of diffuse cholesterosis, they have a width of $0.5-0.8 \mathrm{~mm}$, a length of 3-5mm or more, because they often merge together, forming a kind of mesh (mesh form) (Figure 1a). Comparing with the histological picture of the mucous membrane, it becomes clear that the yellowish formation on the surface is nothing other than the tops of the villi, in the apical part of which are accumulations of foam cells (Figure 1b) translucent through the single-layer epithelium. Back in 1923, Boyed [1] established that the contents of the cytoplasm of these cells are represented by cholesterol esters. Histological and electron microscopic examination of foam cells shows that, depending on the phase of life, the contents of the cell can vary and are structurally quite diverse.

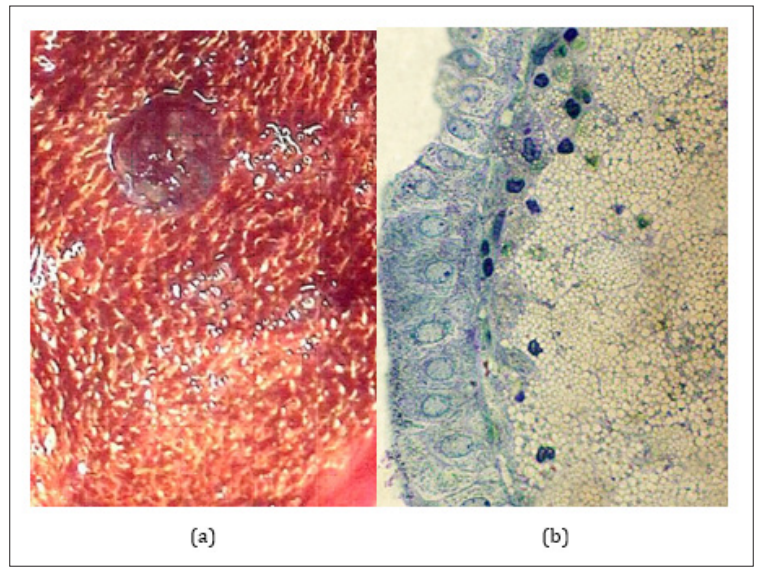

Figure 1: (a) Macroscopic picture of the inner surface of the gallbladder with the classic mesh form of cholesterosis.

(b) Micropolyp site with gallbladder cholesterosis. Cellular nature of cytoplasm foam cells. Semi-thin cut. Coloring - toluidine blue. H. x 1000.

When studying the mucous membrane of the gallbladder in patients with cholesterosis, the main accumulations of foam cells are found in the tops of the villi. When foam cells completely fill the entire space of their own plate, forming the shape of a micropip, the stromal elements almost completely disappear. There is a compression of the capillary bed, which leads to ischemia of the surface epithelium of the mucous membrane and the deterioration of its metabolism. There is a decrease in the height of epithelial cells, the appearance of signs of a decrease in functional activity and, quite often. their desquamation. At the same time, in the neighboring areas of the same villus and in the adjacent villi, the accumulation of foam cells may be substantially less, or not observed at all. In other clinical cases, the spread of the process can go more intensively "and in breadth and depth." Foam cells begin to accumulate intensively in the adjacent apical portions of the villi and in their deeper parts (as if in the neck of a micropolyp) (Figure 2), often reaching before fibromuscular layer. AT the final the result frothy cells of these compartments merge into one whole, forming large cholesterol polyp, visible without any microscope (Figure 3a).

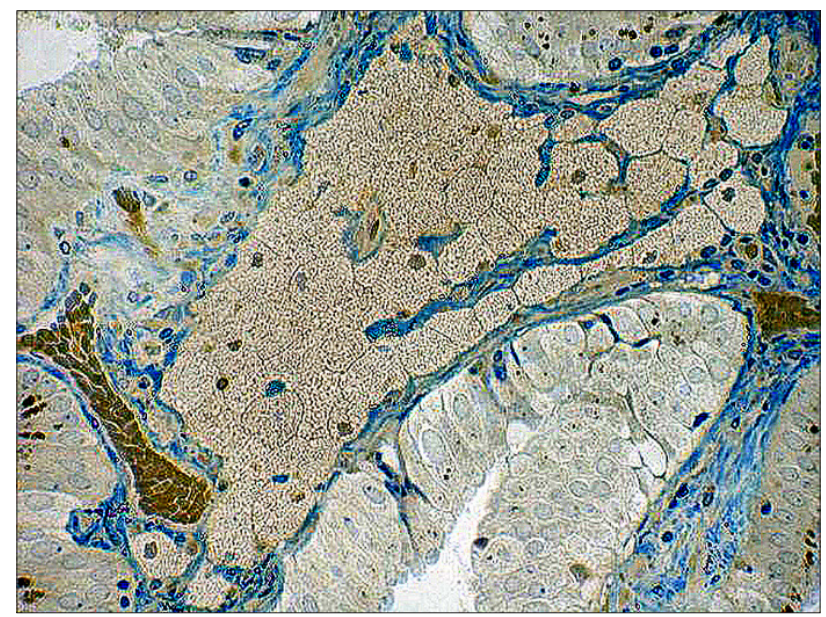

Figure 2:Clusters of foam cells in the neck of the micropip. Semi-thin cut. Coloring - toluidine blue. H. $\mathrm{x} 800$. 


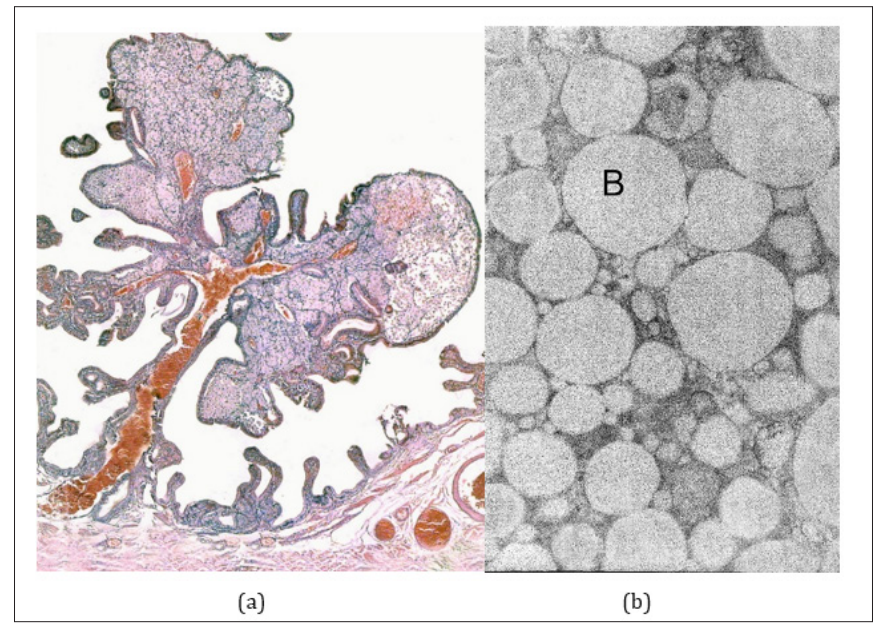

Figure 3: (a) Cholesterol polyp with polypus mesh form of cholesterosis. Stained with hematoxylin and eosin. Increase $\mathrm{x} 40$.

(b) Ultrastructure of vacuoles (c) foam cells (explained in the text). H. x 30000.

The ultrastructure of most foam cells has absolutely no signs of a precursor cell ("phagocyte"). The nucleus of the cell is pyknotic, small in size, with a convoluted karyolemma contour and edge chromatin concentration. In most cases, the entire cytoplasm of the cell is occupied by vacuoles with fine homogeneous content (cholesterol esters), delimited from the cytoplasm by the elemental membrane (Figure $3 \mathrm{~b}$ ). The sizes of these vacuoles vary greatly from small $(0.1-0.2 \mu \mathrm{m})$ to very large $(3 \mu \mathrm{m})$. It is possible that small vacuoles may merge, if we take into account their structural and chemical identity. However, most vacuoles have an average size of 1-1.5 microns. Such cells have no other cytoplasmic inclusions and intracellular organelles, which indicates the absence of any functional activity.

However, in areas of its own plate containing a small number of foam cells, there are cells that retain signs of progenitor cells. In addition to the fact that in them the nucleus has a normal appearance with light karyoplasm and a well-pronounced nucleolus, the cytoplasm contains a small number of cisterns of a rough endoplasmic reticulum (evidence of ongoing intracellular protein synthesis) and phagosomes of various structures. Often these phagosomes contain inclusions, the contents of which structurally highly seem to be on content vacuole frothy cell (Figure 4).

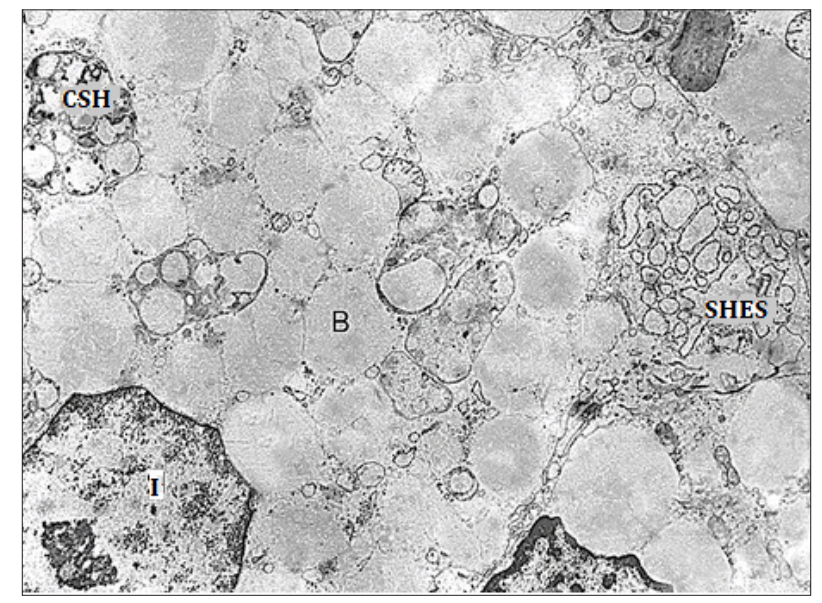

Figure 4: Rough endoplasmic reticulum and cytosegresomes in the cytoplasm of the foam cell. Explanations in the text. Legend: I - the nucleus, SHES - rough endoplasmic reticulum, B - vacuoles, CSG - cytosegresomes. H. $\mathrm{x} 10000$.

It is the last ultrastructural feature (the presence of a large number of phagosomes) that most of all indicates that the macrophage was the precursor of such a foamy cell. The origin of foam cells has long been a debate among scientists. In this regard, those who are engaged in the pathogenesis of atherosclerosis are most active. A small number of researchers studying gallbladder cholesterosis most often rely on these works and cite the results obtained in the study of blood vessels, considering, apparently, that both of these diseases are manifestations of the so-called metabolic syndrome. It is for this reason that the number of cells that can be the source of foamy origin is called adventitial and endothelial cells, macrophages and even smooth muscle cells. 
Podolsky [2] believed that the endothelial cells of lymphatic capillaries are able to accumulate lipids. In addition, they believed that lipids, accumulating in the stroma of the mucous membrane and the lumen of the lymphatic vessels, penetrate (phagocytized auth.) Into the cytoplasm of connective tissue cells and adventitial cells, which contribute to their transformation into foamy cells. About macrophages, as the precursors of foam cells in this book was not indicated. Immunohistochemistry with macrophagespecific anti- CD 68 monoclonal antibodies of several clones was used to determine the histogenetic properties of foam cells in atherosclerosis in humans and in experimental atherosclerosis in animals by several researchers. When cholesterosis of the gallbladder, such studies have not been conducted. But based on the similarity of foam cells in atherosclerotic plaques and the gallbladder, they began to assume that macrophages are precursors of these cells in cholesterosis. However, back in 1990, Beranek [4], using two clones (RAM 11, HAM 56) of macrophage-specific antibodies and antibodies against smooth muscle actin (asm -1, HHF 35) with natural and experimental atherosclerosis, and then in 1997, and our compatriot Andreeva et al. [5], when studying aortic intima, showed very similar pictures, and concluded that aortic intimal smooth muscle cells express macrophage antigens. The authors considered that the mentioned clones of CD 68 antibodies can only be used as a marker of phagocytic cells, but not to study the histogenesis of foam cells. Finally, in 2004 Kunisch et al. [6], using three more clones of anti- CD 68 antibodies (KP11, EBM1, PGM1) for immunohistochemistry and flow cytometry of fibroblasts and endothelial cells in vascular diseases, concluded that CD 68 is not a macrophage-specific antigen. This view was actively supported by the very same Beranek [7].

Starting our immunohistochemical studies of the gallbladder with cholesterosis and knowing about the above-mentioned results obtained in the study of vascular diseases, we, however, first used CD 68 antibodies (clone) to determine the phenotypic properties of foam cells (histogenesis). PGM 1) Kunisch [6] used in 2004. To this we had several considerations. First, in the literature we did not meet the results of immunohistochemical studies performed on the gallbladder with cholesterosis. Secondly, in the mucous membrane of the gallbladder, there are no elements that are present in the vascular intima and are capable of expressing CD 68 antigens, with the exception of lymphocytes and histiocytes (reticular cells according to the old nomenclature).

When analyzing the results of the immunohistochemical reaction with monoclonal CD 68 antibodies, the reaction product was indeed determined in the cytoplasm and on the surface of foam cells, in histiocytes and a certain number of lymphocytes (mainly in the interepithelial position). The illustration below (Figure 5) clearly shows not only the phenotypic identity of foam cells to macrophages, but also the totality of all the above results and considerations: the accumulation of foam cells in the lamina of the top of the villi with micropip formation, their smaller number in the "polyp stem", histiocytes scattered uniformly in the lamina propria (inactivated macrophages), as well as a clear "path" of activated macrophages to the villus peak adjacent to the micropipette.

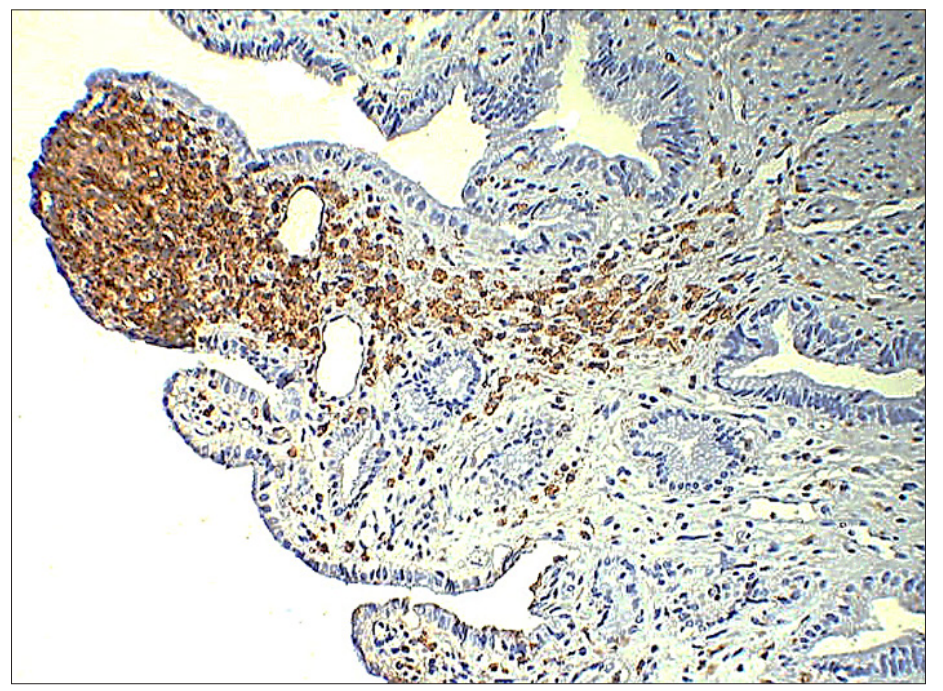

Figure 5: Immunohistochemistry with monoclonal antibodies CD 68 (clone PGM 1). The product of the reaction on the foam cells of micropolip, as well as in the stroma of the villi on the foam cells and histiocytes.

And yet, to finally address the issue of the precursor of foamy cells with gallbladder cholesterosis, we carried out an immunohistochemical study with two new monoclonal macrophage markers - Macrophage Marker NCL-LN 5 and Macrophage Marker NCL-Mac 387. According to the developer's annotation, the listed monoclonal antibodies are most specific for macrophages. The results we obtained using new markers showed that, as with using CD-68, the reaction product was detected in foam cells and in histiocytes of the lamina propria, as well as in activated macrophages in the fibro-muscular layer. The greater specificity of the additionally applied monoclonal antibodies to macrophages (LN 5 and Mac 387) is convinced by the fact that, unlike macrophages, the reaction on lymphocytes in the interepithelial position (T-suppressors) was absent (Figure 6a \& 6b). 


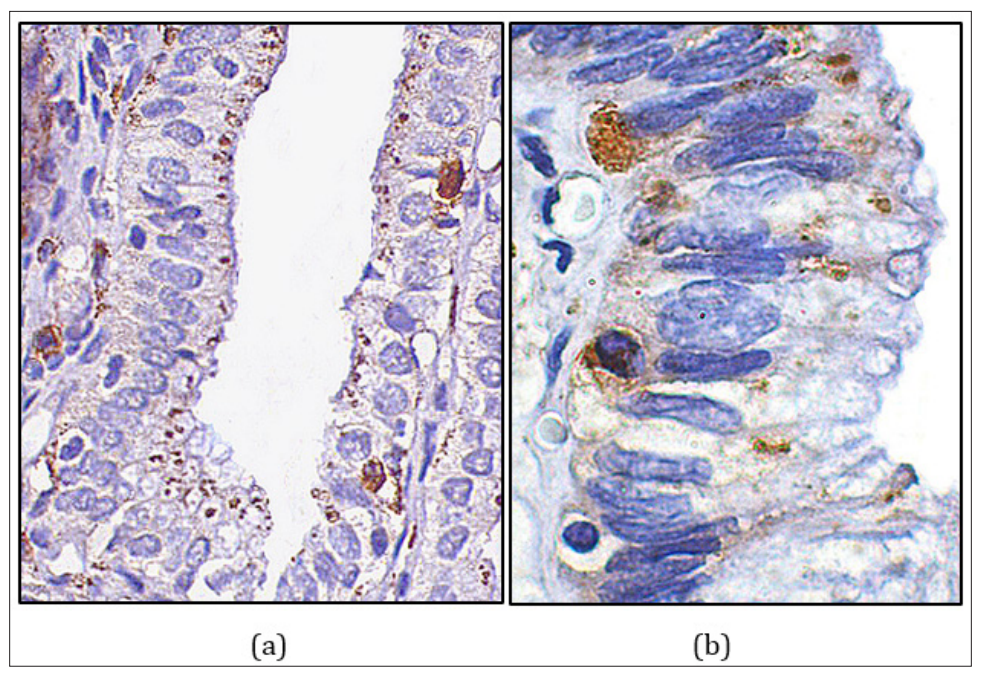

Figure 6: Immunohistochemistry of macrophages in the mucous membrane of the gallbladder. Reaction product on foam cells and its absence on interepithelial lymphocytes.

(a) Clone NCL-LN5 (LN-5), x 200.

(b) Clone NCL-MAC387, x 400.

On the basis of the conducted immunohistochemical studies, the question of the phenotypic precursor of foam cells can be considered solved-it is an activated tissue macrophage. However, one more question remains open: do macrophages of the gallbladder wall have any tissue-specific characteristics or are they the same for macrophages of any organs and tissues? Most authors of publications devoted to cholesterosis, proceed from the fact that the main lipid component, the absorption of which leads to the development of the disease, is cholesterol. It is well known that free cholesterol, which is in the aqueous phase of bile, is absorbed in significant quantities along with water and ions by epithelial cells of the normal mucous membrane [8]. At the same time, the accumulation of cholesterol and its metabolites in the lamina propria of the normal mucous membrane does not occur, because according to physiological laws they do not enter the macrophages, as L considers. Luciano [9], and in the capillary bed of the blood and lymphatic systems and in the general bloodstream. Apparently, the cause of cholesterosis lies in another.

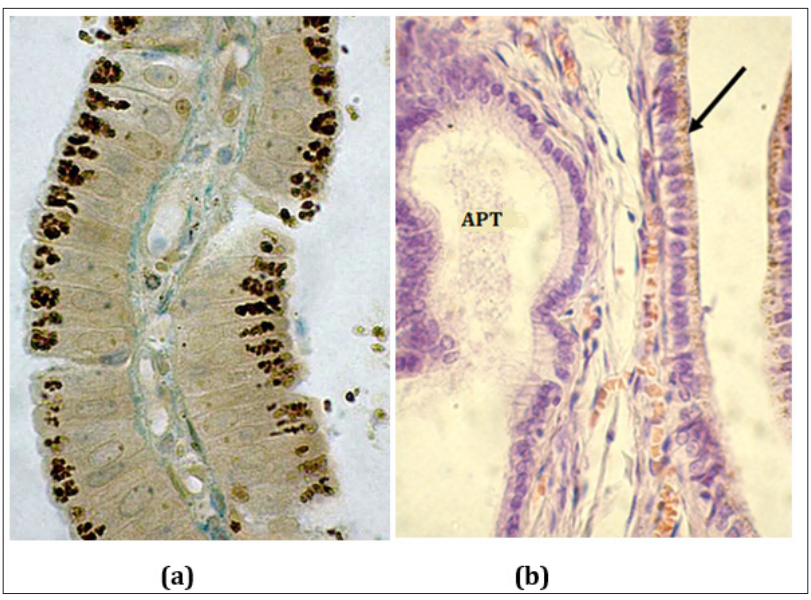

Figure 7: (a) Clusters of osmiophil lipids in the apical region of the absorptive epithelial cells of the mucous membrane of the gallbladder with cholesterosis. Semi-thin section of the osmirovanny drug. Coloring - toluidine blue. H. x 200.

(b) The absorption of lipids by the absorptive epithelium of the villi of the gallbladder (arrow) and the absence of that in the epithelium of the alveolar-tubular glands (APT). H. x 200.

Already at the very beginning of the study of changes in the mucous membrane of the gallbladder with cholesterosis, we noted the presence in the apical part of the absorptive epithelium of fairly large accumulations of lipids. In the study of semi-thin sections of preparations prepared for study in an electron microscope, the inclusions were distinguished by marked osmophilia (Figure 7a). On conventional histological specimens, lipid inclusions were light brown in color. Moreover, they were clearly visible even in unpainted sections. All these characteristics of inclusions testified, first of all, that the lipid structure contains bile pigment - bilirubin, which 
gives a light brown color, and that its presence is associated with the absorption of components of bile, which are not normally absorbed in other pathologies. It was clear that the process of absorption of bile is active, since passive imbibition of the mucous membrane of the bile is not observed. This is clearly seen in the comparison of the morphology of absorptive epithelial cells and a number of secretory cells of the alveolar-tubular glands in the cervical gallbladder, in which lipid transport is not carried out (Figure 7b). In addition, it was noted that the most active absorption of lipids is carried out in areas where there is no accumulation of foam cells in the lamina propria (Figure 8). It is here that activated macrophages appear for the first time, most often in the interepithelial space. In the future, there is a gradual accumulation of macrophages in its own plate, and after all its space is filled with foam cells, the absorptive activity of the epithelium fades, as we wrote above. After we were convinced that such a picture was not only characteristic, but also obligatory with cholesterosis, our data and preliminary considerations were published [10], and the research was continued.

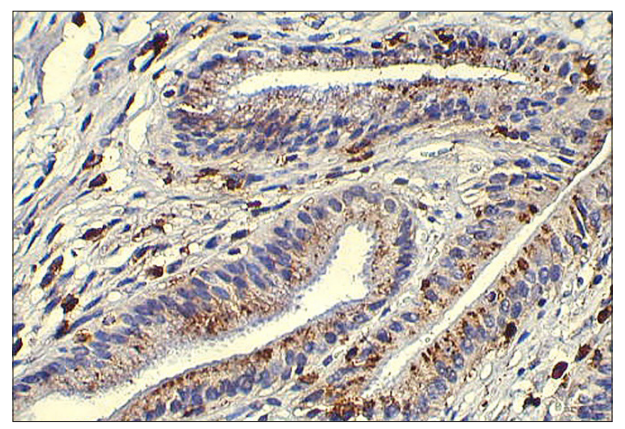

Figure 8: Immunohistochemistry with CD 68 monoclonal antibodies (clone PGM 1). Active absorption of lipids by absorptive cholecites in the area of the villi without foam cells in its own plate. The reaction product on histiocytes.

It was necessary to establish a chain of events that occur in the mucous membrane, starting with the absorption of elements of bile and ending with the accumulation of lipids in the macrophages of the own plate (foam cells). Earlier [11], when studying the absorption of bile in the small intestine outside the digestion period, it was found that the main transport form of bile is bile micelle, which contains bile acids (more precisely bile salts), phospholipids (phosphatidylcholines), proteins and lipoproteins conjugated bilirubin. In electron microscopic examination of surgically removed gallbladders with signs of cholesterosis, in some cases, parietal tissue remains in the preparations. bile not washed from the surface of the mucous membrane as a result of fixation and wiring of the material. At high magnification, it is clearly seen that bile contains osmiophilic bile micelles and triglyceride drops of various sizes (0.1-1.0 microns). Both elements and other elements do not have a limiting membrane (Figure 9a). Apparently, their stability is facilitated by protein molecules located on the surface, which will be discussed below. The true size of the bile micelles can be judged by the measurement results of the most osmiophil micelles, which indicates the passage of the cut plane through their equator. The size of the bile micelles of the cystic bile was found to be about 0.1 microns, which completely coincides with their size in the small intestine. When studying the absorptive epithelium in areas of the mucous membrane outside the accumulation of foam cells (Figure 8 ) in the apical zone of some cells, patterns of endocytic absorption of bile micelles can be found (Figure 9b). It is clearly seen that the number of endocytosis vesicles that do not contain bile micelles (with transparent contents) is significantly larger, which indicates the preserved main (concentration) function of the epithelium. In addition, there is a tendency to merge vesicles containing bile micelles, which is not observed in the small intestine. The lack of rapid movement of the vesicles with bile micelles to the basolateral membrane and their ability to merge with each other leads to the formation of large vacuoles in the supranuclear zone containing bile micelles and small lipid droplets (Figure 10a).

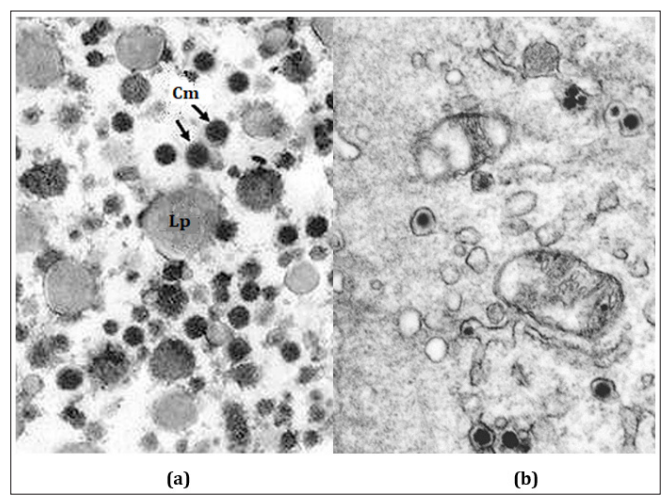

Figure 9: (a) Ultrastructure of cystic bile. Drops of neutral triglycerides (Lp) and bile micelles (Cm). x 50 thousand.

(b) Gall micelles in endocytosis vesicles of absorptive cholecystitis of the mucous membrane of the gallbladder with cholesterosis. x 20 thousand. 


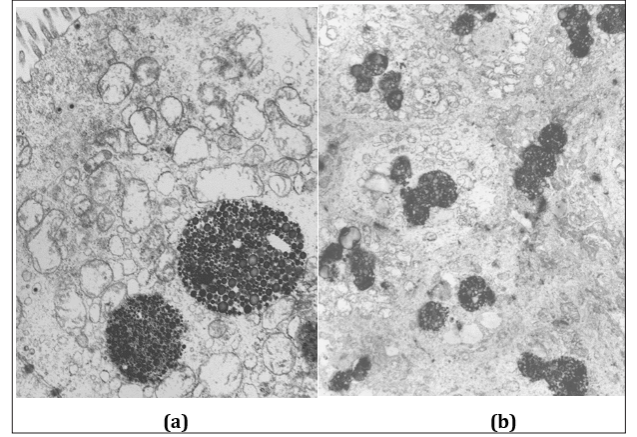

Figure 10: (a) Accumulation of bile micelles in vacuoles of the apical zone of absorptive cholecites of the mucous membrane of the gallbladder with cholesterosis. A few bile micelles in endocytosis vesicles. H. x 8000 .

(b) Numerous vacuoles with bile micelles in a transverse section of the apical region of several absorbent epithelial cells of the gallbladder with cholesterosis. H. x 4000.

Micelles and lipid droplets inside the vacuole do not have a limiting membrane, and the vacuole itself has a very thin elemental membrane that separates its contents from the cytoplasm of the epithelial cell. With electron microscopy, as in a light microscope, it is seen that similar vacuoles are observed in the supranuclear zone of all epithelial cells without exception (Figure 10b). They sometimes merge with lysosomes, resulting in the formation of large cytosegresomes, in which bile micelles and lipids are processed by lysosomal enzymes. However, in most cases, vacuoles with bile micelles move to the lateral membrane (Figure 11) and, merging with it, eject their contents into the inter-epithelial space and then into their own plate. Here, bile micelles become prey of macrophages, which phagocytic them and, after being processed by lysosomal enzymes, are converted into vacuole content characteristic of foam cells (Figure 12a). Some components of bile micelles cannot turn into cholesterol esters and after complete enzymatic processing in lysosomes, they remain until the death of the cell itself in the structure of residual (residual) bodies consisting of lipid droplets, lipofuscin and myelin-like figures (Figure 12b).

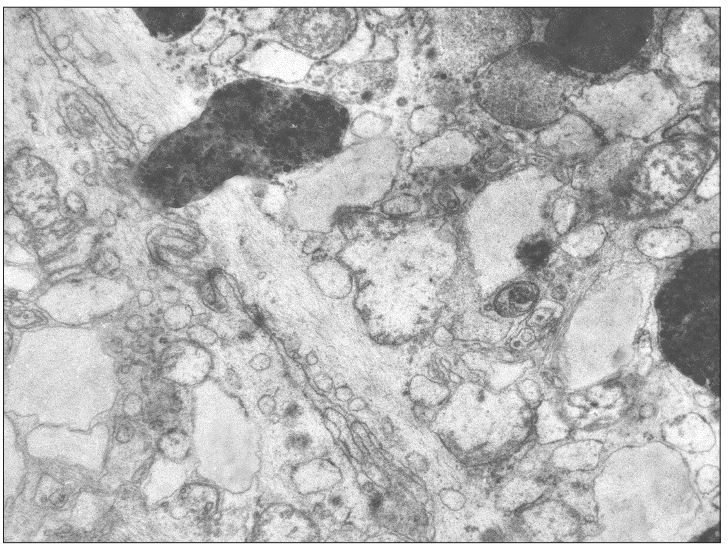

Figure 11: The fusion of vacuoles containing bile micelles with the lateral membrane of the absorptive epithelial cell of the gallbladder with cholesterosis. H. x 15,000 .

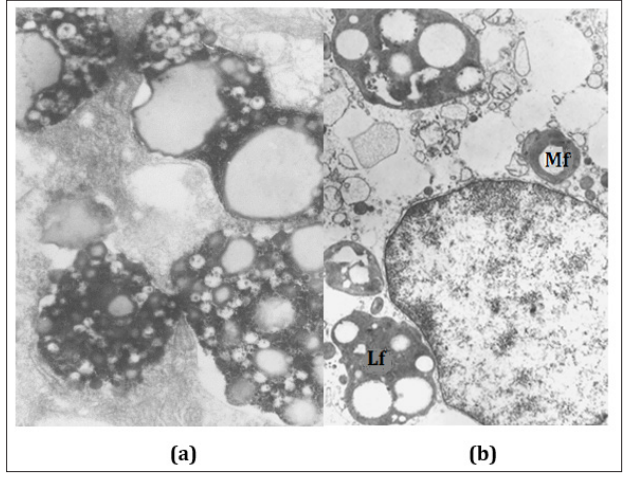

Figure 12: (a) Bile micelles at the stage of hydrolysis and drops of neutral lipids in the structure of secondary lysosomes (cytosegres) of the macrophage of the own plate of the mucous membrane of the gallbladder with cholesterosis. $\mathrm{H}$. $\mathrm{x} 15,000$.

(b) Residual bodies - myelin-like figures (Mf) and lipofuscin (Lf) in a foamy cell of the mucous membrane of the gallbladder with cholesterosis. $\mathrm{H}$. x 10,000 .

It should be noted that in the literature we have met only one publication of the 1975 Todo [12]. with co-authors [12], in which in one case out of eight studied gallbladders with cholesterosis, the electron microscopic method described the accumulation of epithelial cells of digitonin-positive circular formations (bile micelles) and plate - like structures in the supranuclear zone (lipid drops-auth.), which did not give a reaction with digitonin (histochemistry of cholesterol). Both of these structures did not have a limiting membrane. The authors concluded that the epithelial cells of the gallbladder with cholesterosis contain a lot of cholesterol, which forms the structures described as a result of cholesterol precipitation during rapid fixation. After 10 years, one of the authors of the previous work of Koga [13]. published an article [13], in which an analysis of the study of the same eight cases of cholesterosis of the gallbladder was presented more accurately. Using light and electron microscopy, the accumulation of lipid inclusions was again noted, but already in the agranular reticulum (!?) Of the supranuclear epithelial cell zone. Lipid inclusions were also found in the macrophages of the lamina propria, which turned into foam cells. The author noted that these cells are regular and cannot pass through the endothelium of the lymphatic vessels, or large foam cells cause obstruction and further destruction of these vessels. After another 12 years, the same Koga [13]. together with Satoh [14] re-published the work, but in another journal, which completely repeated the previous one, both in content and in conclusions. Apparently from the description of the results received by Koga [13]. and his colleagues, the pictures they observed coincide with those that we received in our study. However, the interpretation of Japanese scientists based on the knowledge and hypotheses of the time does not explain the pathogenetic mechanisms of cholesterosis. During the period 70-80 of the last century, the endocytosis (pinocytosis) transport process was well known, and Cheikerd and Palard (1962) even observed pinocytosis of fat by epithelial cells after its introduction into the guinea pig gall 
bladder. Nevertheless, the transmembrane absorption mechanism dominated in the minds of scientists. Even observing with an electron microscope micelles containing cholesterol, Japanese scientists explain their pro and convergence of precipitation by the action of the retainer, and bile micelles in endocytotic vesicles issue of lipid inclusions in the smooth endoplasmic reticulum, which is more typical of the secretory m cells s, not the suction epithelium. This work proved macrophage-derived foam cells, although the debate on this issue extends to the present time.

What are the reasons that lead to changes in the properties of bile micelles and the acquisition of characteristics that ensure their absorption by the epithelium of the gallbladder? One of the possible causes of structural and chemical changes in bile is the nutrition and chemical composition of the lipid components of the diet. Numerous experiments on animals, conducted in the 60-70s of the last century, indicate that excessive consumption of cholesterol with food entails an increase in its level not only in blood serum, but also in bile. And although some have expressed doubts about the importance of cholesterol-rich bile for the development of cholesterosis, the excessive consumption of animal fats always negatively affects the cardiovascular and biliary systems. It would be very useful to obtain information on the prevalence of cholesterosis among vegetarians, but no such studies have been conducted. However, there is information [15] on the positive effect of vegetarianism on the lipid spectrum of blood serum, which is one of the causes of the lower mortality from coronary heart disease of vegetarians compared to the general population. For example, in California, in a survey of 27,530 Adventists, it was found that mortality from myocardial ischemia in vegans is $77 \%$ lower than in non-vegetarians. In addition, cholesterosis can be observed not only in humans and is peculiar, apparently predominantly carnivorous. For example, while browsing the Internet with educational illustrations for classes and exams for students on this topic on the websites of universities in the USA and Europe, we found on the University website of Virginia Health System photo of the structure of the normal mucous membrane of the gallbladder of a dog, on which there are signs of cholesterosis in the form of foam cells in its own plate and even micropipes (Figure 13).

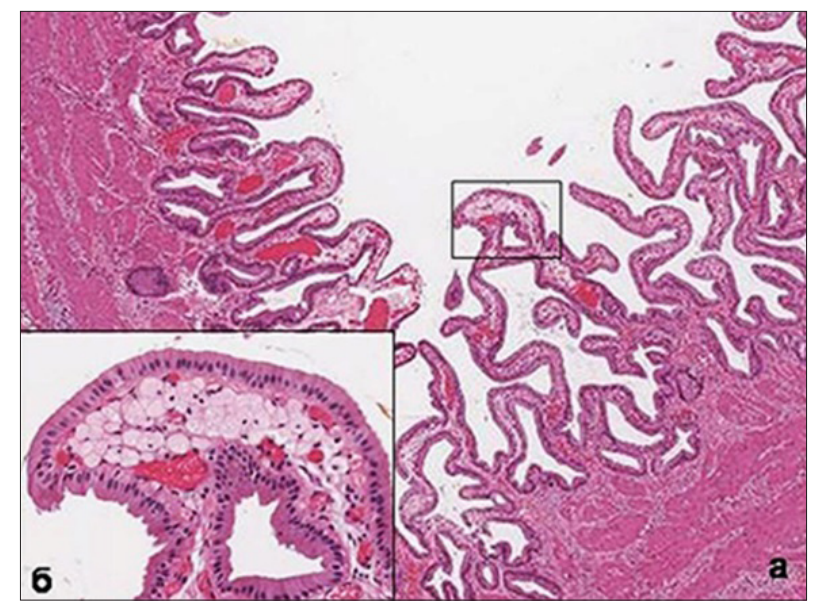

Figure 13: Signs of cholesterosis of the gallbladder in a healthy dog.

(a) The accumulation of foam cells in the apical areas of the villi of the mucous membrane. H. x 200.

(b) Fragment of the apical part of the villus with foamy cells. H. x 800 .

Sources of cholesterol esters in foam cells can serve up to $80 \%$ of the components of bile micelles containing cholesterol. Under normal conditions, already in the process of synthesis in the hepatocyte, bile acids are conjugated with glycine and taurine in a ratio of 3: 1, forming primary salts. In some types of liver pathology, as well as under the influence of nutritional and hormonal factors, this ratio may change in favor of taurine conjugates. Considering their higher polarity (compared to glycine salts) [16], we can expect changes in the physicochemical properties of bile micelles, the main component of which (up to 70\%) are conjugated bile acids. An equally important factor that affects the Physio-chemical properties of bile, is the change in the ratio of bile acids and cholesterol. When cholesterosis occurs on the background of normal cholesterol in the blood serum, its content in bile increases by 2-3 times, mainly due to low-density lipoprotein cholesterol [17], while the amount of bile acids decreases by the same amount. Not only solubilizing and transport properties of bile micelles are reduced, but also their stability. As a result, part of the bile micelles can obtain properties that ensure their endocytic absorption by the epithelium of the gallbladder. Apparently, only some part (possibly small) of bile micelles acquires these properties, and the disease develops extremely slowly before the onset of clinical manifestations. Currently, there is no methodological ability to isolate bile from the common pool and obtain the physicochemical characteristics of that part of the bile micelles that are capable of endocytosis transport by the epithelial cell.

Proteins, which make up about $4.5 \%$ of the weight of bile micelles, are an extremely important component of them. In bile, there are several dozen proteins with a molecular weight of from 6 to $220 \mathrm{kDa}$. The bulk of the proteins are globulins, and the smaller blood albumin, which by endocytosis is captured on the sinusoidal membrane of the hepatocyte and, after transcytosis, 
is released by exocytosis into the bile capillaries. A small part of the bile proteins is synthesized in the hepatocyte and also enters the bile. Already in the lumen of the bile capillaries at the time of the formation of bile micelles, part of the proteins (apoA, apoB, apoC, apoE and their various phenotypes) are embedded in their surface, which is why they are called apoproteins. Apoproteins are osmotically active components. ApoA-I is supposed to function as a cholesterol solubilizer [18], while apoB determines the quantitative relationship between cholesterol and phospholipids in bile [19]. Normally, the apoA: apoB ratio varies between 3-3.5: 1. RI Ivanchenkova [20] found that the number of apoB in the cystic bile with cholesterosis decreases, and the ratio of apoA-1 to apoB becomes maximum $(\approx 8: 1)$. How this phenomenon can affect the Physio-chemical properties of bile micelles remains incomprehensible. However, bile proteins play an extremely important role in the pathogenesis of cholesterosis. Of all the components of bile, only proteins (like antigens), which penetrate into their own epithelial plate as part of bile micelles, can trigger activation and attraction of tissue macrophages (histiocytes) into the "zone of conflict". Other components of bile are lipid in nature and do not cause the immune system to respond.

A fairly frequent combination of cholesterosis of the gallbladder with atherosclerosis and hypertension (63.4-83.5\%) suggests that the general pathogenesis of these diseases is based on general disorders of lipid metabolism. The key organ regulating these processes is the liver, which synthesizes the elements of bile, and also forms its transport form. The resulting imbalance between the amount of conjugated bile acids reduced by a factor of 2-3 and LDL cholesterol increased by as many times leads to the disruption of the colloidal balance of a part of the bile micelles and their ability to endocytosis by the epithelium of the mucous membrane of the gallbladder. A further chain of events unfolding directly in the gallbladder wall is described above. Thus, the pathogenesis of cholesterosis of the gallbladder consists of successive stages. At the first stage, as a result of a violation of the synthetic function of hepatocytes, qualitative changes occur in the lipid and lipoprotein components of the cell membranes and the composition of the forming bile. At the second stage, the modified biliary micelles are absorbed by absorbing cells of the biliary epithelium, followed by enzymatic catabolism and the formation of cholesterol esters released into the intercellular space of its own plate. At the third stage, cholesterol metabolites circulating in their own plate become prey to tissue macrophages, which absorb them but are not capable of their further utilization. This leads to the formation of the phenotype of a foam-stand with their accumulation among the structures of the gallbladder wall and the macroscopic manifestation cholesterosis.

\section{References}

1. Boyed W (1923) Studies in gallbladder pathology. Br J Surg 10(39): 337 356.

2. Permyakov NK, Podolsky AE (1969) Cholesterosis of the gallbladder. Medicine, p. 103.

3. Ilchenko AA, Morozov IA, Khomeriki SG, Orlova YN (2007) Cholesterosis of the gallbladder. GEOTAR, Russia, p. 232.

4. Beranek JT, Cavarocchi NC (1990) Smooth muscle cells and macrophages in rabbit cardiac allograft atherosclerosis. J Heart Transplant 9(6): 732.

5. Andreeva ER, Pugach IM, Orekhov AN (1997) Subendothelial smooth muscle cells of human aorta express macrophage in situ and in vitro. Atherosclerosis 135(1): 19-27.

6. Kunisch E, Fuhrmann R, Roth A (2004) Macrophage specificity of three anti-CD68 monoclonal antibodies (KP1, EBM11, and PGM1) widely used for immunoh i stochemistry and flow cytometry. Ann Rheum Dis 63(7): 774-784.

7. Beranek JT (2005) CD68 is not a macrophage-specific antigen. Ann Rheum Dis 64(2): 342-344.

8. Jacyna M, Ross P, Bacar M (1987) Characteristics of cholesterol absorption by human gallbladder: Relevance to cholesterosis. J Clin Pathol 40(5): 524-529.

9. Luciano L (1989) Morphological aspects of cholesterol storage in the human gallbladder. Prog Clin Biol Res 295: 269-275.

10. Morozov IA, Khomeriki SG, Ilchenko AA, Orlova YN (2005) Ultrastructural changes in the mucous membrane of the gallbladder during the formation of cholesterosis. Hepatology 5: 20-22.

11. Morozov PA, Lysikov luA, Khvylya SI (1985) Electron microscopic study of the process of hepato-intestinal recycling of the components of bile. Fiziol Zh SSSR Im I M Sechenova 71(11): 1419-1427.

12. Koga A, Todo S, Nishimura M (1975) Electron microscopic observation of the gallbladder cells. Histochemistry 44(4): 303-306.

13. Koga A (1985) Fine structure of the human gallbladder with cholesterosis with special reference to the mechanism of lipid accumulation. Br J Exp Pathol 66(5): 605-611.

14. Satoh H, Koga A (1997) The structure of cholesterolosis in the human gallbladder and the mechanism of lipid accumulation. Microsc Res Tech 39(1): 14-21.

15. Medkova I. Vegetarianism and diseases of the century, p. 6.

16. Reshetnyak VI (2003) Mechanisms of bile formation and primary biliary cirrhosis. Publishing House "Red Square", Moscow, Russia, p. 142.

17. Slivka OY (1981) Gallbladder cholesterosis. Lab Delo 11: 653-656.

18. Kibe A, Holzbach R, LaRusso N (1987) Human low-density lipoprotein and biliary lipid secretion. Gastroenterol 92: 382-390.

19. Kawamoto T, Mao SJ, La Russo NF (1987) Biliary excretion of apolipoprotein B by the isolated perfused rat liver. Relationship to receptor-mediator uptake of human low-density lipoprotein and biliary lipid secretion. Gastroenterol 92 (5 Pt 1): 1236-1242.

20. Ivanchenkova RA (2006) Chronic diseases of the biliary tract. M Atmosphere, p. 416

For possible submissions Click below: 